\title{
Opening Editorial
}

This Special Issue of the European Journal of Risk Regulation is the result of an international workshop held in January 2013 in Paris, at the initiative of the French Agency for Food, Environmental and Occupational Health \& Safety (ANSES) and the Center for the Sociology of Organisations (CSO). Following a survey conducted in 2011 regarding the use of human and social sciences in fifteen public organisations in Europe and North America in charge of risk assessment and/or risk management related to environmental and health issues, an interdisciplinary group of researchers and practitioners from seven countries met to discuss the results of this survey and to compare experiences in the use of social sciences in country case studies. It was agreed to pursue this initiative by launching a network (known provisionally as the "Paris Risk Group"). This network comprises social scientists and representatives of various organisations involved in food safety, occupational health and safety, and environmental health in various European countries. The network's overall goal is to promote the use of social sciences alongside more traditional scientific disciplines by sharing experiences on the use of social sciences in the production of risk assessments and in the broader context of risk management, as well as by sharing methods, tools and experiences across organisations.

Interest for the use of social sciences in risk assessment and management stems from major institutional reforms undertaken over the last two decades by national and European public health authorities confronted with a multiplication of controversies, crises and scandals on issues related to food safety, occupational health and safety, and environmental health. The reforms were undertaken in order to improve organisational ability to govern complex issues such as genetically modified organisms, nanotechnology, endocrine disruptor compounds or more broadly chemicals in food, the environment or the workplace. Overall, the creation of specialised risk assessment organisations in charge of producing science-based evidence prior to decision-making led to a significant improvement in risk regulation.

Yet, there remains room for progress. On many issues, namely those mentioned above, the science-base for risk assessment often remains largely inconclusive, in particular with respect to long-term adverse health effects. There is thus a growing recognition that these uncertain risks need specific and renewed governance processes, both from a risk assessment and a risk management perspective. Alongside more "traditional" forms of scientific knowledge (essentially from natural sciences), which is applied in current frameworks of risk assessment or risk analysis, it has become clear that many of the above-mentioned issues required bringing together other forms of knowledge. This is necessary in order to capture, to characterise and to evaluate the many uncertainties these issues are fraught with; and to gain a better understanding of the perception and handling of these risks by different stakeholders and the broader public. This can also serve to highlight risk regulation strategies and the consequences of action or inaction, whether driven by public policy or private initiative. 
Among these other forms of knowledge, social sciences have a specific contribution: they can provide useful knowledge in improving the risk assessment process itself; they can also offer insights to help adapt the process to more complex, ambiguous and uncertain issues. This explains the growing importance that social sciences and humanities have progressively acquired in various risk assessment and/or management organisations; along with the variety of approaches according to the remit and field of expertise of these organisations. There is, here again, room for progress in order to encourage the uses social sciences and humanities as there is no "one size fits all". Therefore, sharing experiences and drawing lessons from concrete case studies where expertise in social sciences is called for is a way forward in the recognition of social sciences as an integral part of the risk governance process.

The four papers that compose this Special Issue illustrate different contributions of social sciences to the risk assessment and management of environmental and health issues.

The introductory paper by Cécile Wendling provides a general overview of experiences in bringing social scientists to work in, or for, risk assessment and risk management organisations in four European and two North-American countries. The paper starts off by recalling the bulk of knowledge now available in social sciences on health and environmental risks, along with a general acknowledgement by the different actors in charge of risk assessment and management of the importance of these disciplines in understanding risk. Wendling then shows that behind the great variety of experiences collected, the results are strikingly similar: they suggest a limited incorporation of these forms of knowledge in expertise and decision-making. She suggests a set of explanations for this: social sciences are time-consuming and resource-intensive; social sciences are a source of greater complexity, when the process is designed to simplify an already complex issue; social sciences tend to blur the border between science and politics, when the process is designed to establish a clear boundary between these two dimensions. In the end, Wendling proposes several ways of improving the current situation; all depend on the willingness and capacity of heads of agencies and policymakers to introduce social sciences in risk assessment and management.

The three other papers present specific contributions by the social sciences. These go beyond the traditional contribution of social sciences to risk analysis (risk perception and communication) and suggest innovative ways of incorporating social sciences in risk assessment and management

The paper by Yannick Barthe relates the experience of a social scientist embedded in a group of French experts commissioned to produce a report on the risks of electromagnetic fields. While initially asked to provide a synthetic view of studies in the social sciences on the issue of EMF, his attention quickly shifts to an assessment of expert procedures on controversial issues. He reveals the many ambiguities that characterize these procedures, and how the staging of an independent, transparent and collective endeavour leads to a fragmented expertise, in which the experts' values and prefer- 
ences are never openly discussed, yet play a determinant role in their evaluation of the risk. This leads to a controversy, when the head of the agency formulates recommendations that are considered inconsistent with the conclusions of several leading experts in the group, who feel betrayed and publicly denounce a political interpretation of their scientific advice. Yet, Barthe insists, this results from a confusion by these experts between science and expertise. Expertise is an intermediate form of knowledge between science and policy, with distinct rules regarding the production of evidence. Furthermore, in the case of controversial issues, expertise must sometimes grant uncertainties extra weight in the process, if only to make sure that decisions resting solely on validated (but often limited) scientific results do not ultimately lead to type 2 errors.

Keune et al. in their paper relate a different experience, in which social sciences are called upon to organise an "analytical deliberative decision support procedure" on human biomonitoring in Flanders. The intervention of social scientists follows the recognition by experts and policymakers that the move from scientific expertise to decisionmaking on a highly sensitive issue is not easily achieved, given that the evaluation of the scientific results requires forms of judgment that are not strictly professional. This leads to a series of procedural devices designed to help evaluate the evidence in order to offer advice to the government. These consist in particular in the inclusion of stakeholders, along with a variety of experts. The final result is a complex, but robust process of evaluation, resting both on the diversity of knowledge forms and viewpoints introduced in the process, and original methods to organize, test and discuss these. Yet in the end, the authors conclude that, although a success, this initiative will not be easily replicated given the complexities entailed, in particular from a methodological point of view, and the necessary efforts by the different partners to make this work. In a sense, this remains a prototype.

Benamouzig et al. suggest another approach. Based on an extended cooperation with Anses, they propose a sociological checklist destined to help cover and assess the various dimensions of issues the agency is asked to work on. Starting with the premise that most issues cannot be restricted to scientific questions, the checklist suggests a way of identifying and exploring the other dimensions, while still providing a useful tool for the agency to provide policymakers with recommendations. The authors identify 6 dimensions (institutional context; socioeconomic context; social practices and context; problem construction; forms of knowledge; social inequalities) whose exploration may help, not only to better understand the nature and underlying dynamics of the issue at hand, but also to provide an assessment that ultimately takes into account the variety of expectations, questions and demands amongst the different stakeholders. Apart from its contribution to a more robust risk assessment, this checklist also organizes in a clear manner the various forms of knowledge produced by the social sciences during the last three decades on environmental and health risks.

In the end, all four papers suggest that a great deal of effort is still needed in promoting the contribution of social sciences to risk assessment and management. The great- 
est challenge remains the demonstration that this contribution, if it is indeed time-consuming and a source of added-complexity in the initial stages, ultimately saves time by avoiding that the reports and advices produced by risk assessment and management organizations are immediately contested on the grounds that they are incomplete or biased. But two other challenges exist. As Wendling points out, the contribution of social sciences tends to fall somewhere in between the mythical distinction between science and policy, assessment and management. In a sense, it blurs the "boundary work" that so many actors have undertaken in order to clearly distinguish these two stages. Paradoxically, while most actors consider that this boundary does not hold in reality, they are still committed to it as it provides a convenient way of shielding themselves from unwanted influences and critics. Hence, the contribution of social sciences is seen as potentially troubling, as it openly questions the standard process of risk analysis. The second challenge is identified by Barthe when he points out that social sciences are different from other sciences in two ways. First, they produce statements that cannot be simply considered as true or false, but are rather "modes of investigating reality which are sound, to a greater or lesser degree." Second, within social sciences coexist different theoretical and epistemological approaches: hence they will never provide a unified viewpoint but rather a kaleidoscopic representation. Traditional experts and policymakers may see this as problematic. Yet it offers a more realistic representation of risk issues. The problem then becomes one of organizing and evaluating the knowledge available: the papers by Keune et al. and Benamouzig et al. offer two different methods to that end. There are certainly many others.

Olivier Borraz - CNRS Research professor - Centre for the Sociology of Organisations (Sciences Po-CNRS) Benoit Vergriette - Head of Risks and Society Unit - ANSES 